Early Initiated Postoperative Rehabilitation Reduces Fatigue in Patients with Operable Lung Cancer

\title{
A Randomized Trial
}

Quist, M.; Sommer, M. S.; Vibe-Petersen, J.; Bohlbro, M. Staerkind; Langer, S.; Larsen, K.; Trier, K.; Christensen, M.; Clementsen, P.; Missel, M.; Henriksen, C.; Poulsen, K.; Langberg, H.; Pedersen, J.

\section{Published in:}

Journal of Thoracic Oncology

DOI:

10.1016/j.jtho.2018.08.256

Publication date:

2018

Document version

Publisher's PDF, also known as Version of record

Document license:

Unspecified

Citation for published version (APA):

Quist, M., Sommer, M. S., Vibe-Petersen, J., Bohlbro, M. S., Langer, S., Larsen, K., Trier, K., Christensen, M., Clementsen, P., Missel, M., Henriksen, C., Poulsen, K., Langberg, H., \& Pedersen, J. (2018). Early Initiated Postoperative Rehabilitation Reduces Fatigue in Patients with Operable Lung Cancer: A Randomized Trial. Journal of Thoracic Oncology, 13(10), S329. https://doi.org/10.1016/j.jtho.2018.08.256 
results support the effects of the early rehabilitation intervention on enhancing oxygenation, preventing complications, and promoting recovery from lung cancer surgery as indicated by shortened the duration of chest tube drainage. Surgery to remove the cancer is one of the primary treatment options for non-small cell lung cancer. However, lung cancer surgery may result in decreasing lung capacity and expansion; therefore, increase risks for postoperative pulmonary complications. Pulmonary rehabilitation designed to enhance lung expansion and ventilation may help to reduce postoperative lung complications and promote patients' recovery from lung cancer surgery. Keywords: pulmonary rehabilitation, lung cancer surgery, oxygenation

\section{OA04.06 \\ Perceptions of Non-Participation in a Rehabilitation Intervention After Surgery for Non-Small Cell Lung Cancer}

\section{Schoenau, ${ }^{1}$ M. Missel ${ }^{2}{ }^{1}$ Department of Thoracic Surgery,} University Hospital of Copenhagen, Rigshospitalet, Copenhagen/DK, ${ }^{2}$ Department of Thoracic Surgery, University Hospital of Copenhagen, Copenhagen/DK

Background: Patients with non-small lung cancer (NSCLC) are difficult to engage in clinical trials. Few studies have examined in-depth why these patients refuse to participate. In a Danish randomized clinical trial; 'Postoperative rehabilitation in operable lung cancer patients (PROLUCA)' only $32 \%$ of eligible participants consented to participate in the trial. The purpose of this qualitative study was therefore to explore perceptions, considerations and barriers of non-participation in PROLUCA. Method: This study was inspired by Reflective Life Research as developed by Dahlberg et al. as a descriptive and interpretive phenomenological research approach. Participants are patients who declined to participate in PROLUCA (nonparticipants). They were purposefully sampled and recruited from the group of patients who were found to be eligible for the exercise intervention but who declined to participate. Data were collected though telephone interviews. Openness, curiosity and sensitivity played an important role in carrying out the interviews. Analysis was performed according to Reflective Life Research. Result: Fifteen non-participants consented to participate in qualitative interviews. Nine men and six women with a mean age of 68 years (range 48-84) were included. Mean time since surgery was 21 month (range 12-28). Five patients were working and ten were retired, eleven patients lived with a partner. The analysis revealed three essential themes referred to the patients' experiences of being 'Between healthy life and good life', 'Under the influence of society' and their experiences of 'Health and rehabilitation as a personal responsibility'. Perceptions of non-participation in rehabilitation after surgery for lung cancer are moderated between freedom and necessity. Patients experience ambivalence between a wish to participate in rehabilitation and not having the energy to participate. Patients refused to participate due to daily life priorities and lack of motivation which furthermore is related to social and interpersonal relationships. The patients exercise history is also essential in declining participation. Additionally the patients are under influence of norms and health perceptions from the society. Conclusion: Patients' perception of "the good life" was fundamental for accepting or declining participation in a rehabilitation intervention study. Consideration and barriers of non-participation was influenced by norms from the society, motivation, priorities, exercise history, social and interpersonal relations. This study has contributed with a sensitive awareness of why patients following lung cancer surgery might refuse participating in rehabilitation. This knowledge can be taken into consideration in the planning of future clinical trials with lung cancer patients. Keywords: Surgery, Rehabilitation, Nonparticipation

\section{OA04.07}

Early Initiated Postoperative Rehabilitation Reduces Fatigue in Patients with Operable Lung Cancer: A Randomized Trial

M. Quist, ${ }^{1}$ M.S. Sommer, ${ }^{2}$ J. Vibe-Petersen, ${ }^{2}$ M. Stærkind Bohlbro, ${ }^{1}$ ${ }_{\text {S. Langer, }}{ }^{3}$ K. Larsen, ${ }^{4}$ K. Trier, ${ }^{2}$ M. Christensen, ${ }^{5}$ P. Clementsen, ${ }^{6}$ M. Missel, ${ }^{5}$ C. Henriksen, ${ }^{7}$ K. Poulsen, ${ }^{5}$ H. Langberg, ${ }^{8}$ J. Pedersen ${ }^{9}$ ${ }^{1}$ University Hospitals Centre for Health Research, Department 9701, Rigshospitalet, University of Copenhagen, Copenhagen $/ D K,{ }^{2}$ Copenhagen Centre for Cancer and Health, Copenhagen Municipality, Copenhagen/DK, ${ }^{3}$ Dept. of Oncology, Rigshospitalet, Copenhagen $\emptyset / D K,{ }^{4}$ Dept. of Respiratory Medicine, Bispebjerg University Hospital, Copenhagen Nv/ DK, ${ }^{5}$ Dept. of Thoragic Surgery, University Hospital of Copenhagen, Copenhagen/DK, ${ }^{6}$ Medicine, Zealand University Hospital, Roskilde/DK, ${ }^{7}$ Social Medicin, Copenhagen University, Copenhagen $/ D K,{ }^{8}$ Copenhagen Healthtech Cluster, Copenhagen/DK, ${ }^{9}$ Cardiothoracic Surgery Rt 2152, Rigshospitalet, Copenhagen University, Copenhagen/DK

Background: Surgical tolerability and perioperative risk of complications are correlated with high age, smoking history, comorbidities, low cardiorespiratory fitness $\left(\mathrm{VO}_{2}\right.$ peak) and low functional capacity, which paradoxically are characteristics describing the average patient with lung cancer. Little is known about the optimal amount and timing of exercise strain in concern of the operation wound and with regard improvement of physical function and quality of life (QOL). On this background, we decided to investigate the effect of early vs. late initiated postoperative rehabilitation in patients with operable lung cancer on exercise capacity, functional capacity, muscle strength, and QOL. Method: The study was designed as a two-armed randomized controlled trial with randomization to either early initiated postoperative rehabilitation (14 days after surgery (ERG)) or a control arm with late initiated postoperative rehabilitation (14 weeks after surgery (LRG)). The primary endpoint was a change in maximum oxygen consumption (VO2peak) from baseline to post intervention 26 weeks following lung resection. Fatigue was measured with EORTC QLQ C30 LC13. Result: From April 2013 to June 2016, 582 patients with operable NSCLC were screened for eligibility. With 119 patients randomized in the early rehabilitation group (ERG) (68 females, 51 males; median age 65), and 116 randomized to late rehabilitation group (LRG) (62 females, 54 males; median age 65) the recruitment rate was $52.6 \%$. There was a non-significant decrease in $\mathrm{VO}_{2}$ peak in both ERG and LRG from baseline to 26 weeks and no significant difference between ERG and LRG ( $p=0.9269)$. There was a significant decrease from baseline to 14 weeks in both ERG $(\mathrm{p}=0.027)$ and LRG $(p<0.001)$ and a significant difference between groups $(p=0.0018)$ There was a non-significant increase from 14 weeks to 26 weeks in ERG $(p=0.464)$ and a significant increase from 14 weeks to 26 weeks in LRG $(p<0.001)$ and a significant difference between the two groups $(\mathrm{p}=0.0003)$. We found no significant differences in QOL but we found a significant difference between ERG and LRG from baseline to 14 weeks in fatigue level in favour of ERG. Conclusion: This is the first randomized controlled trial to investigate the effects of early vs. late initiated postoperative rehabilitation in patients with lung cancer There is no difference in the commencement (early vs. late) of a postoperative exercise program for patients with lung cancer on exercise capacity. But to reduce fatigue patients should be recommended to initiate early exercise programs. Keywords: post surgery rehabilitation, fatigue 